\title{
A SHORT PROOF OF A GENERATING FUNCTION FOR JACOBI POLYNOMIALS
}

\author{
DENNIS STANTON ${ }^{1}$
}

\begin{abstract}
A short proof is given for Bailey's bilinear generating function for Jacobi polynomials. It depends only upon the orthogonality relation for Jacobi polynomials and a quadratic transformation for a hypergeometric series. A $q$ analog is also stated.
\end{abstract}

Bailey [4, equation (2.1)] found the following bilinear generating function for Jacobi polynomials:

$$
\begin{aligned}
\sum_{n=0}^{\infty} \frac{n !(\alpha+\beta+1)_{n}}{(\alpha+1)_{n}(\beta+1)_{n}} P_{n}^{(\alpha, \beta)}(x) P_{n}^{(\alpha, \beta)}(y) t^{n} \\
=(1+t)^{-\alpha-\beta-1} F_{4}((\alpha+\beta+1) / 2,(\alpha+\beta+2) / 2 ; \alpha+1, \beta+1 ; z, Z) \\
=(1+t)^{-\alpha-\beta-1} \sum_{n, m=0}^{\infty} \frac{(\alpha+\beta+1)_{2(m+n)}}{n ! m !(\alpha+1)_{n}(\beta+1)_{m}}\left[\frac{t}{4(1+t)^{2}}\right]^{m+n} \\
\quad \cdot(1+x)^{m}(1+y)^{m}(1-x)^{n}(1-y)^{n},
\end{aligned}
$$

where $z=t(1+x)(1+y) /(1+t)^{2}, Z=t(1-x)(1-y) /(1+t)^{2}$, and $(a)_{n}=$ $\Gamma(a+n) / \Gamma(a)$. He used Watson's transformation, which expresses an $F_{4}$ as the product of two ${ }_{2} F_{1}$ 's. In this paper we give a short proof of (1) which uses only the orthogonality of Jacobi polynomials and a quadratic transformation for a generalized hypergeometric series.

The Jacobi polynomials $\left\{P_{n}^{(\alpha, \beta)}(x)\right\}_{n=0}^{\infty}$ are orthogonal on $[-1,1]$ with respect to the weight function $w(x)=(1-x)^{\alpha}(1+x)^{\beta}, \alpha, \beta>-1$. We take the usual normalization, $P_{n}^{(\alpha, \beta)}(1)=(\alpha+1)_{n} / n !$, so that $P_{n}^{(\alpha, \beta)}(-x)=(-1)^{n} P_{n}^{(\beta, \alpha)}(x)$. Askey [3] has shown how to obtain Jacobi's generating function for $P_{n}^{(\alpha, \beta)}(x)$ from the orthogonality relation. The same idea works for the bilinear generating function.

Let $G(x, y, t)=\sum_{n=0}^{\infty} a_{n}(x, y) t^{n}$ be the right-hand side of (1). It is clear that $a_{n}(x, y)$ is a polynomial of degree at most $n$ in both $x$ and $y$. For any polynomials $q_{k}(x)$ and $r_{l}(y)$ of degrees $k$ and $l$, consider

$$
I(t)=\int_{-1}^{1} \int_{-1}^{1} G(x, y, t)(1-x)^{\alpha}(1+x)^{\beta} q_{k}(x)(1-y)^{\alpha}(1+y)^{\beta} r_{l}(y) d x d y .
$$

A necessary condition for $a_{n}(x, y)=b_{n} P_{n}^{(\alpha, \beta)}(x) P_{n}^{(\alpha, \beta)}(y), b_{n} \neq 0$, is that $I(t)$ is a polynomial in $t$ of degree $\min (k, l)$. This follows immediately from the orthogonality relation for $P_{n}^{(\alpha, \beta)}$. However this condition is also sufficient. To see this, let

Received by the editors November 19, 1979.

AMS (MOS) subject classifications (1970). Primary 33A65, 33A30; Secondary 42 A56.

${ }^{1}$ The author was partially supported by NSF Grant MCS-78-18222. 


$$
a_{n}(x, y)=\sum_{i, j=0}^{n} b_{i j}^{n} P_{i}^{(\alpha, \beta)}(x) P_{j}^{(\alpha, \beta)}(y) .
$$

By successively choosing $k, l=0,1, \ldots, n$ we have that $b_{i j}^{n}=0$ unless $i=j=n$. This proof works for any set of orthogonal polynomials with a positive weight function $d \alpha(x)$ which has an infinite number of points of increase.

To prove (1) we put $q_{k}(x)=(1+x)^{k}$ and $r_{l}(y)=(1-y)^{l}$. The integrals are evaluated by beta functions to obtain

$$
\begin{aligned}
I(t)= & A(1+t)^{-\alpha-\beta-1} \sum_{m, n=0}^{\infty} \frac{(\alpha+\beta+1)_{2(m+n)}(\beta+k+1)_{m}(\alpha+l+1)_{n}}{m ! n !(\alpha+\beta+k+2)_{m+n}(\alpha+\beta+l+2)_{m+n}} \\
& \cdot\left[\frac{t}{(1+t)^{2}}\right]^{n+m}
\end{aligned}
$$

where $A \neq 0$ is independent of $t$. Putting $n=N-m$, and summing the resulting $m$ sum by Vandermonde's theorem [5, p. 3], we have

$$
\begin{aligned}
I(t)= & A(1+t)^{-\alpha-\beta-1} \sum_{N=0}^{\infty} \frac{(\alpha+\beta+1)_{2 N}(\alpha+\beta+l+k+2)_{N}}{N !(\alpha+\beta+k+2)_{N}(\alpha+\beta+l+2)_{N}} \\
& \cdot\left[\frac{t}{(1+t)^{2}}\right]^{N} \begin{array}{cc}
(\alpha+\beta+2) / 2, \\
\alpha+\beta+k+2, \\
=
\end{array} \\
\alpha(1+t)^{-\alpha-\beta-1} F_{2}\left(\begin{array}{c|c}
(\alpha+\beta+1) / 2, k+l+2 \\
\alpha+\beta+l+2
\end{array}\right. & \left.\frac{4 t}{(1+t)^{2}}\right) .
\end{aligned}
$$

However there is a quadratic transformation for a well-poised ${ }_{3} F_{2}[5$, p. 97]

$$
\begin{aligned}
& (1+t)_{3}^{-a} F_{2}\left(\begin{array}{ccc|c}
a / 2, & (a+1) / 2, & 1+a-b-c \mid & 4 t \\
1+a-b, & 1+a-c & (1+t)^{2}
\end{array}\right) \\
& ={ }_{3} F_{2}\left(\begin{array}{cc}
a, & b, \\
1+a-b & c \\
1+a-c
\end{array} \mid-t\right),
\end{aligned}
$$

Thus we have for $a=\alpha+\beta+1, b=-l, c=-k$,

$$
I(t)=A_{3} F_{2}\left(\begin{array}{cc}
-k, & -l, \\
\alpha+\beta+2+k, & \alpha+\beta+2+l
\end{array} \mid-t\right),
$$

which is clearly a polynomial in $t$ of degree $\min (k, l)$. To evaluate the constants $b_{n}$ put $x=1$ and $y=-1$ to obtain

$$
\sum_{n=0}^{\infty} b_{n} P_{n}^{(\alpha, \beta)}(1) P_{n}^{(\alpha, \beta)}(-1) t^{n}=(1+t)^{-\alpha-\beta-1} .
$$

Since $P_{n}^{(\alpha, \beta)}(1)=(\alpha+1)_{n} / n$ ! and $P_{n}^{(\alpha, \beta)}(-1)=(\beta+1)_{n}(-1)^{n} / n$ ! we obtain (1).

Unfortunately we were not directly led to (1). However, we can write down a $q$-analog of equation (1) by this technique. We take the little $q$-Jacobi polynomials $P_{n}(x, a, b ; q)$ as defined in [1, equation (3.1)]. Carlitz has proved a $q$-analog of (4) 
[6]. (Although his proof works only for $a=q^{-k}$, the formula remains true as a power series in $t$ for any $a$.) The result is

$$
\begin{aligned}
& \sum_{n=0}^{\infty} \frac{(a b q)_{n}(a q)_{n}}{(q)_{n}(b q)_{n}}(b q)^{n} q^{\left(\frac{n}{2}\right)} P_{n}(x ; a, b, q) P_{n}(y ; a, b, q) t^{n} \\
& =\frac{(-a b q t)_{\infty}}{(-t)_{\infty}} \sum_{n, m=0}^{\infty} \frac{(a b q)_{2(n+m)}(b q x)_{m}(b q y)_{m}\left(x b y q^{2}\right)^{n} q^{m}}{(q)_{m}(q)_{n}(a q)_{n}(b q)_{m}(-a b q t)_{n+m}(-q / t)_{n+m}}
\end{aligned}
$$

where $(a)_{n}=(1-a)(1-a q) \cdots\left(1-a q^{n-1}\right)$.

A $q$-analog of the Poisson kernel for Jacobi polynomials [2, equation (2.32)] is

$$
\begin{gathered}
\sum_{n=0}^{\infty} \frac{(a b q)_{n}(a q)_{n}}{(q)_{n}(b q)_{n}}\left(1-a b q^{2 n+1}\right)(b q)^{n} q^{\left(\frac{n}{2}\right)} P_{n}(x ; a, b, q) P_{n}(y ; a, b, q) t^{n} \\
=\frac{\left(-a b q^{3} t\right)_{\infty}}{(-t)_{\infty}}\left(1-a b q^{2} t^{2}\right)(1-a b q) \\
\cdot \sum_{n, m=0}^{\infty} \frac{\left(a b q^{2}\right)_{2(n+m)}(b q x)_{m}(b q y)_{m}\left(x y b q^{2}\right)^{n} q^{m}}{(q)_{m}(q)_{n}(a q)_{n}(b q)_{m}\left(-a b q^{3} t\right)_{n+m}(-q / t)_{n+m}}
\end{gathered}
$$

This is not the Poisson kernel for $q$-Jacobi polynomials because there is no $q^{\left(\frac{n}{2}\right)}$ factor in the $L^{2}$-norm [1, Theorem 9].

The same technique proves the Hille-Hardy formula for Laguerre polynomials and Mehler's formula for Hermite polynomials.

\section{REFERENCES}

1. G. Andrews and R. Askey, Enumeration of partitions: the role of Eulerian series and q-orthogonal polynomials, Higher Combinatorics (M. Aigner, Ed.), Dordrecht, Boston, Mass., 1977, pp. 3-26.

2. R. Askey, Orthogonal polynomials and special functions, CBMS Regional Conf. Ser. in Appl. Math., vol. 21, SIAM, Philadelphia, Pa., 1975.

3. __ Jacobi's generating function for Jacobi polynomials, Proc. Amer. Math. Soc. 71 (1978), 243-246.

4. W. Bailey, The generating function of Jacobi polynomials, J. London Math. Soc. 13 (1938), 8-12.

5. ___ Generalized hypergeometric series, Cambridge Univ. Press, Cambridge, 1935.

6. L. Carlitz, Some formulas of F. H. Jackson, Monätsh. Math. 73 (1969), 193-198.

Department of Mathematics, Massachusetts institute of Technology, Cambridge, MasSACHUSETTS 02139

Current address: School of Mathematics, University of Minnesota, Minneapolis, Minnesota 55455 